force estimation, piezoelectric actuators, machine tool deflection,

online compensation

\author{
Eckart UHLMANN ${ }^{1,2}$ \\ Mitchel POLTE ${ }^{1,2}$ \\ Florian TRIEBEL ${ }^{1 *}$ \\ Rasmus OVERBECK ${ }^{1}$ \\ Simon THOM $^{1}$
}

\title{
ACCURACY IN FORCE ESTIMATION APPLIED ON A PIEZOELECTRIC FINE POSITIONING SYSTEM FOR MACHINE TOOLS
}

\begin{abstract}
In order to improve the accuracy of machine tools, the use of additional active modules meeting the requirements of the "Plug \& Produce" approach is focused. In this context one approach is the installation of a high precision positioning table for online compensation of machine tool deflections. For the model-based determination of the deflection, the knowledge of the effecting process force is crucial. This article examines the use of displacement sensors for force estimation in a piezoelectric system. The method is implemented on a high precision positioning table applicable in milling machine tools. In order to compensate nonlinear effects of piezoelectric actuators, a hysteresis operator is implemented. Experimental investigations are carried out to quantify the influence of preload stiffness, preload force and workpiece weight. Finally, a resolution $\mathrm{d} \leq 78 \mathrm{~N}$ could be achieved and further improvements to meet the requirements for online compensation of machine tool deflection are discussed.
\end{abstract}

\section{INTRODUCTION}

The working accuracy of machine tools is affected by static, dynamic and thermal deformations as well as tribological changes. Thereby, the static stiffness $k_{\text {stat }}$ is an important characteristic of machine tools. It is directly related to the working accuracy of machine tools, since loads resulting from net weight and process forces $F_{p}$ lead to deformation of the structure and thus to displacement $\delta_{\text {tcp }}$ of the Tool Center Point (TCP) [1]. To compensate the machine tool deflection the Institute for Machine Tools and Factory Management (IWF) of the Technische Universität Berlin focuses on the development of a modular add-on compensation unit. This unit should follow the "Plug \& Produce" approach, which is described as the capability of production systems to automatically integrate components into the running system without manual efforts and changes [2]. Therefore, the challenge of the

\footnotetext{
${ }^{1}$ Institute for Machine Tools and Factory Management IWF, TU Berlin, Germany

${ }^{2}$ Institute for Production Systems and Design Technology IPK, Fraunhofer, Germany

*E-mail: triebel@iwf.tu-berlin.de https://doi.org/10.36897/jme/134002
} 
integration into machine tools with different control systems arises. The goal of this paper is to enable a piezoelectric compensation unit to measure effecting process forces $F_{p}$ without the use of force sensors. For that, the force estimation is applied and the achievable resolution $d$ is determined.

\subsection{COMPENSATION OF TOOL-CENTER-POINT DISPLACEMENT}

There are two different procedures for active compensation of displacement $\delta_{\text {tcp }}$ at the TCP caused by process forces $F_{p}$. Offline compensation is performed before actual machining. Habibi et al. [3] propose a procedure, which includes the calculation of occurring process forces $F_{p}$ for each machining point in the preprocessing. This data is used as input for a model of machine deformation and finally a compensated tool path is generated via an adapted $\mathrm{NC}$ code.

The second method for active compensation of displacement $\delta_{\text {tcp }}$ caused by process forces $F_{p}$ is online compensation. This type of compensation is based on measured values of process forces $F_{p}$ and takes place in real time during the machining process. Compared to offline compensation the advantages are the independence from a model for the calculation of process forces $F_{p}$, the feasibility to react on unexpected events and the saved time by not pre-processing the NC code. However, machine tool integrated sensor technology is necessary for measurement of process forces $F_{p}$ and the model for determining displacement $\delta_{\text {tcp }}$ must be real-time capable. Various approaches for online compensation are available through research work. Denkena et al. [4] propose an approach where the process force $F_{p}$ is measured with a dynamometer and the displacement $\delta_{\text {tcp }}$ is calculated via experimentally determined stiffness $k$ of the machine tool as well as calculated stiffness $k$ of the milling tool. The compensation of displacement $\delta_{\text {tcp }}$ is done by a spindle attachment, which allows fine positioning by using parallel kinematics consisting of piezoelectric actuators. A slightly different approach uses process forces $F_{p}$ recorded by the dynamometer as an input variable for an analytical model of deflection $x_{T}$ of the milling tool [5]. This model defines the milling tool as a bending beam and the resulting displacement $\delta_{\text {tcp }}$ is compensated by a separate fine positioning unit. Another concept determines process forces $F_{p}$ via spindle head integrated strain gauges [6]. The displacement $\delta_{\text {tcp }}$ is calculated on basis of the experimentally determined stiffness $k$. Compensation of displacement $\delta_{\text {tcp }}$ takes place via inherent feed axes of the machine tool by transferring the real-time calculated displacement $\delta_{\text {tcp }}$ to the control system of the machine tool.

The presented methods for online compensation are based either on the use of inherent feed axes of the machine tools or additional redundant ones. The use of inherent feed axes requires an intervention in the control system of machine tools and thereby is not suitable for "Plug \& Produce" regarding different machine tools. Redundant fine positioning drives with a high resolution can be realized using piezoelectric actuators. Positive characteristics of piezoelectric actuators include handling of high force $F_{\text {piezo }}$, quick response time $t_{r}$, high acceleration $a$ and high stiffness $k$ as well as a very compact design [7]. However, the limitation in travel, occurring hysteresis and sensitivity to tensile loads must be considered. 


\subsection{SELF-SENSING}

The principle known as self-sensing describes the ability of piezoelectric actuators to determine physical states such as deflection $x_{\text {piezo }}$ or applied force $F_{\text {piezo }}$ without use of external sensors [8]. Values are determined by using signals, which are generated by the piezoelectric actuator itself and can be measured with relatively little effort. Eliminating the need for additional sensors, cost and space savings are achieved [9]. However, nonlinear effects of the piezoelectric actuators such as hysteresis and creep must be handled by this method.

There are different approaches for "self-sensing" that use either the piezoelectric effect or the changes in electrical properties of the actuators [8]. The use of the piezoelectric effect is based on the reversible correlation between an applied force $F_{\text {piezo }}$ and the generation of an electric field $E$ caused by electrical charges $q$. In practice, a distinction is made between methods of measuring voltage $U_{\text {piezo }}$ and electrical charge $q$. For approaches based on the measurement of voltage $U_{\text {piezo }}$, hysteresis is relevant. Therefore, the use of a hysteresis operator is recommended $[9,10]$. The second method uses the linear relationship between applied electric charge $q$ and deflection $x_{\text {piezo }}$ of the actuator [11]. The electric charge $q$ is determined by measuring incoming and outgoing currents $I$. Since the applied voltage $U_{\text {piezo }}$ itself induces electrical charges $q_{\text {ind }}$, evaluation electronics are necessary to separate the self-induced electric charge $q$ ind from the applied electric charge $q$ [8].

When using the electrical properties of the actuator for self-sensing, the change of permittivity $\varepsilon[12]$ or capacity $C[13,14]$ is measured. This is realized by superimposing the applied voltage $U_{\text {piezo }}$ with a high-frequency voltage $U_{H}$ with small amplitude $A$, which causes detectable currents $I_{H}$, but does not lead to a deflection $x_{\text {piezo }}$ of the actuator. Using this approach, a hysteresis-free determination of the capacity $C$ can be performed [13] and the relationship between permittivity $\varepsilon$ and deflection $x_{\text {piezo }}$ can be described as linear [12].

\section{ACTIVE ERROR COMPENSATION UNIT}

At the IWF, the active compensation unit shown in Fig. 1, was developed as an add-on for outdated machine tools to increase the working accuracy [15]. This is achieved by compensating geometrical errors. Until now, displacement $\delta_{\text {tcp }}$ caused by process forces $F_{p}$ is not considered by the compensation unit.

While the three inherent feed axes $(x, y, z)$ of the machine tool are used for coarse positioning, the active compensation unit takes over fine positioning in $x$ - and $y$-direction by use of two redundant axes. These are driven by piezoelectric actuators of the type PSt 1000/25/125 VS35 made by Piezosystem Jena GmbH, Jena, Germany. Main com-ponent of the active compensation unit is a monolithic structure made of aluminum AW 7075, which is used due to its high yield strength $\sigma_{y}$ and low Young's modulus $E$, so that the used flexure hinges can be subjected to high stress. To reduce cross-coupling effects of two axes, the monolithic structure contains decoupling elements. The contact between the piezoelectric actuator and the decoupling element is achieved by a spherical-shell-arrangement. 


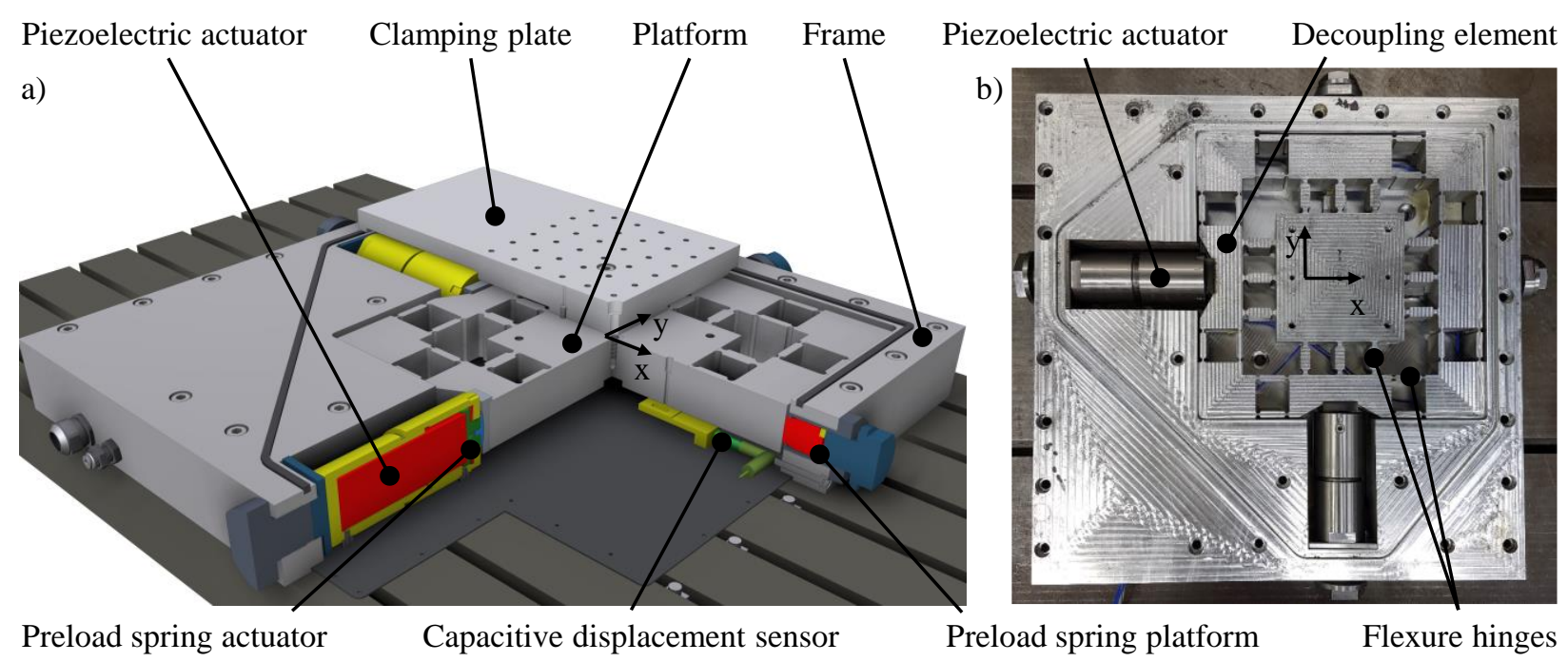

Fig. 1. Active compensation unit: a) CAD model, b) top view

The piezo-electric actuators are pretensioned by springs, so that even in dynamic operation only compressive forces $F_{\text {comp }}$ have impact on the piezoelectric actuators. The platform is also pretensioned by springs to allow the returning motion of the platform towards the actuators. To measure the displacement $x_{p}$ of the platform, the active compensation unit is equipped with capacitive displacement sensors of the type CS1 made by Micro-Epsilon Mess-technik GmbH \& Co. KG, Ortenburg, Germany. The compensation unit is controlled by a control board of the type DS1103 made by dSPACE GmbH, Paderborn, Germany.

\section{GENERAL APPROACH}

Aim of the investigation provided in this paper is to determine the accuracy of estimation of applied process force $F_{p}$ with the compensation unit presented in Section 2 without use of external force sensors. The approach for estimating applied process forces $F_{p}$ differs from the previously presented self-sensing approaches in Section 1.2. The compensation unit is equipped with high-precision displacement sensors, which eliminate estimation of deflecttion $x_{\text {piezo }}$ of the piezoelectric actuators. Furthermore, information about the displacement $x_{p}$ of the platform opens the opportunity to estimate the applied process forces $F_{p}$ without knowledge of electrical charges $q$. In all following investigations voltage $U_{\text {piezo }}$ is controlled and displacement $x_{p}$ of the platform is measured. Applying an external force $F$ results in a deformation $\delta_{\text {piezo }}$ of the piezoelectric actuator and thereby cause a parallel shift of its hysteresis curve in vertical direction. Figure 2 shows two exemplary hysteresis curves of a piezoelectric actuator corresponding to a voltage profile altering between a minimum and a maximum voltage $U_{\text {piezo. }}$. The magnitude of deformation $\delta_{\text {piezo }}$ can be calculated with the stiffness $k_{\text {piezo. }}$

$$
\delta_{\text {Piezo }}=F / k_{\text {piezo }}
$$


An evaluation of the deformation $\delta_{\text {piezo }}$ allows to draw a conclusion about the applied force $F$. However, the force estimation is affected by hysteresis of the piezoelectric actuators. Therefore, the resolution $\mathrm{d}$ of the force estimation corresponds to the product of maximum height $h_{\max }$ of the hysteresis curves and stiffness $k_{\text {piezo }}$ of the piezoelectric actuators or, in case of the compensation unit stiffness $k_{p}$ of the platform, see Section 4.

$$
d=k_{p} \times h_{\max }
$$

- Hysteresis curve

- - Hysteresis curve under load F

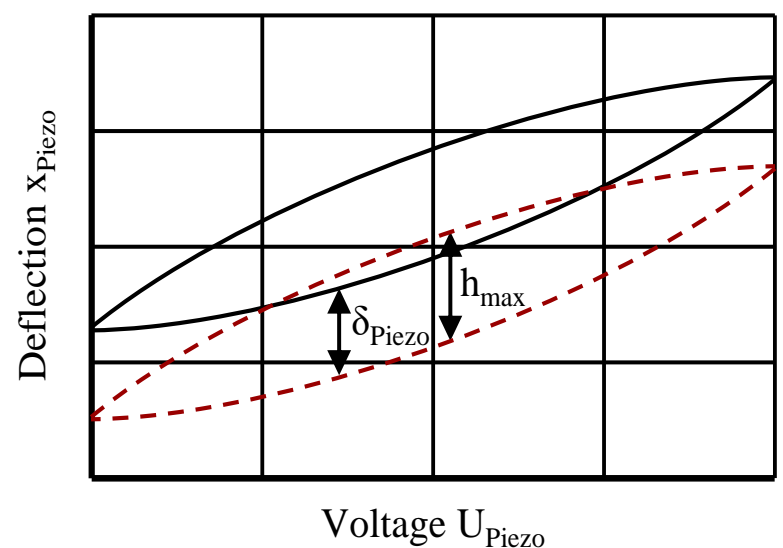

F : Applied external force

$\delta_{\text {Piezo }}:$ Deformation of piezoelectric actuator caused by external force $\mathrm{F}$

$\mathrm{h}_{\max }$ : Max. height of hysteresis curve

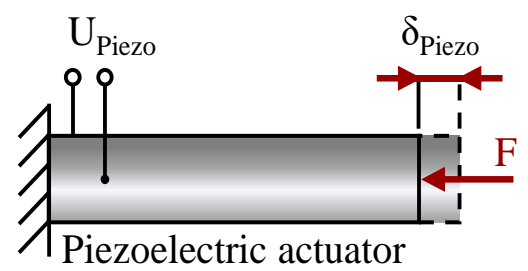

Fig. 2. Hysteresis curve of a piezoelectric actuator

One possibility to reduce resolution $d$ is the use of a hysteresis operator. The operator estimates the current hysteresis of the actuator, which then can be eliminated from the calculation. As a result, resolution $d$ is no longer determined by maximum height $h_{\max }$ of the hysteresis curve, but by maximum error $e_{\max }$ between estimated and real hysteresis curve.

$$
d=k_{p} \times e_{\max }
$$

Equation 3 shows that an improvement of the resolution d can either be achieved by reducing the stiffness $k_{p}$ or the maximum error $e_{\max }$. Besides deviation resulting from inaccuracy of the hysteresis operator, the error $e_{\max }$ includes disturbances as noise of the sensors and creep of the piezoelectric actuators.

\section{IMPLEMENTATION}

The approach presented in Section 3 is implemented on the compensation unit. Due to the symmetrical structure of the compensation unit, the following examinations focus on one axis. It is necessary to take a closer look at the stiffness $k_{p}$ of the platform of the compensation unit. Therefore, a substituted mechanical system of the compensation unit is created, where the influence of all partial stiffnesses is shown. 


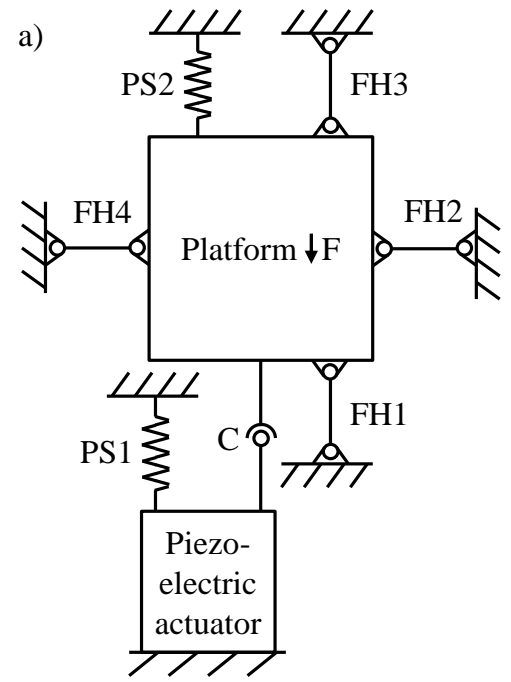

$\mathrm{k}:$ Stiffness

$\mathrm{F}$ : Effecting force

$$
\begin{aligned}
& \text { PS : Preload spring } \\
& \text { C : Connection between } \\
& \text { actuator and platform } \\
& \text { FH : Flexure hinge }
\end{aligned}
$$

b)

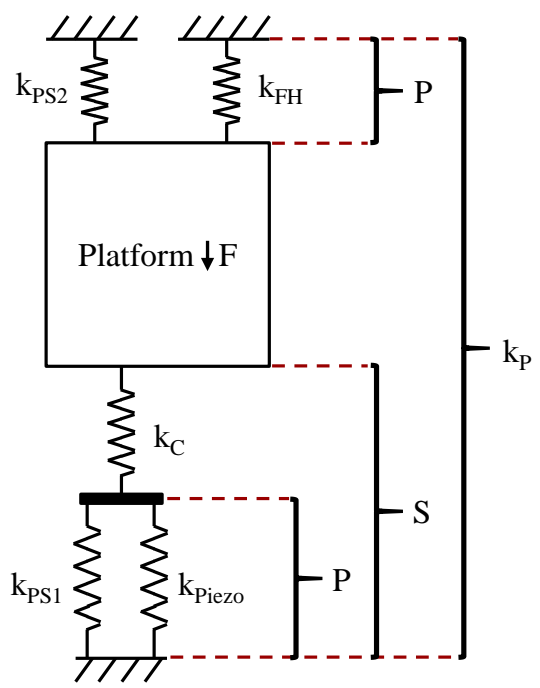

P: Parallel connection

$S$ : Serial connection

Fig. 3. Determination of stiffness $k_{p}$ of the platform of the compensation unit: a) schematic overview; b) spring model

Figure 3 a shows a schematic overview of the compensation unit. The platform is supported by four flexure hinges, which can be summarized to the stiffness $k_{\mathrm{FH}}$. A preload spring protects the actuator against tensile load. A further preload spring pushes the platform towards the actuator. The connection between actuator and platform is represented by the stiffness $k_{c}$. Figure $3 \mathrm{~b}$ shows a spring model of the compensation table. Beside the preload springs PS1 and PS2 all elements, i.e. actuator, flexure hinges and the connection between actuator and the platform, are approximated by springs. This results in the following Equation 4 for the stiffness $k_{p}$ of the platform.

$$
k_{p}=k_{\mathrm{FH}}+k_{\mathrm{PS} 2}+\frac{\left(k_{\text {piezo }}+k_{\mathrm{PS} 1}\right) \times k_{\mathrm{c}}}{k_{\text {piezo }}+k_{\mathrm{PS} 1}+k_{\mathrm{c}}}
$$

\subsection{DETERMINATION OF THE STIFFNESS}

The stiffness $k_{\mathrm{PS} 1}$ and $k_{\mathrm{PS} 2}$ of the preload springs and the stiffness $k_{\text {piezo }}$ of the piezoelectric actuator are specified in data sheets. The stiffness $k_{p}$ of the platform and the stiffness $k_{\mathrm{FH}}$ of the flexure hinges are unknown and therefore are determined experimentally as shown in Fig. 4. The stiffness $k_{c}$ of the connection between actuator and platform can be calculated afterwards.

A horizontal force $F_{H}$ is applied to the compensation unit via an eyebolt, a pulley and a pull weight. The force $F_{H}$ leads to a displacement $x_{p}$ of the platform, which is detected by internal displacement sensors, so that the stiffness $k_{p}$ of the platform can be determined:

$$
k_{p}=\frac{F_{H}}{X_{P}}
$$




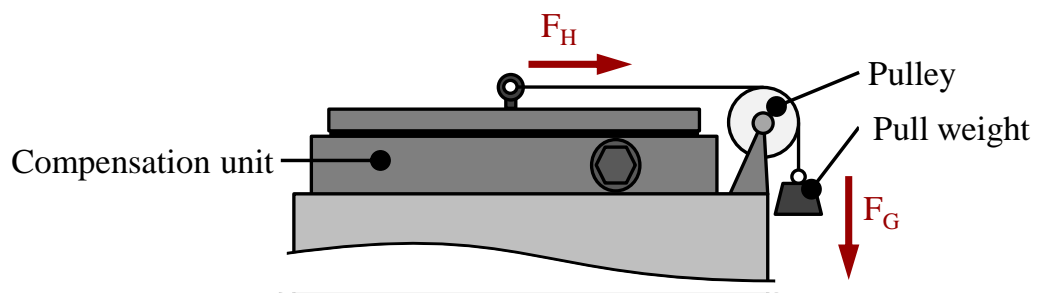

Fig. 4. Experimental setup for determination of stiffness of compensation unit

The determination of the stiffness $k_{\mathrm{FH}}$ of the flexure hinges is performed analogically, with removed actuators and preload springs PS1 and PS2. Table 1 shows an overview of all partial stiffnesses of the compensation unit including the calculated stiffness $k_{c}$.

Table 1. Stiffnesses of the compensation unit

\begin{tabular}{|l|c|}
\hline Stiffness & Value $[\mathrm{N} / \mu \mathrm{m}]$ \\
\hline$K_{p}$ & 155 \\
\hline$K_{\text {piezo }}$ & 300 \\
\hline$k_{\mathrm{PS} 1}$ & 10 \\
\hline$k_{\mathrm{PS} 2}$ & 12 \\
\hline$k_{\mathrm{FH}}$ & 37 \\
\hline$k_{c}$ & 161 \\
\hline
\end{tabular}

\subsection{EXPERIMENTAL ERROR ANALYSIS}

Besides the stiffness $k_{p}$, the resolution $d$ depends on the maximum error $e_{\max }$ between estimated and measured hysteresis curve. For the estimation of the hysteresis curve, a Prandtl-Ishlinskii operator is implemented. A mathematical description is provided by Rakotondrabe [16]. According to Ivan et al. [10] and Rakotondrabe et al. [9] the PrandtlIshlinskii operator is particularly suitable for the estimation of piezoelectric hysteresis curves. Subsequently, the influences of the preload force FPF, the preload stiffness $k_{\mathrm{PS} 2}$ and the mass $m_{w}$ of a workpiece on the size of the maximum error $e_{\max }$ are experimentally investigated. These are influences, which can be changed at idle, but not during operation.

The model is considered as quasi-static, so only excitations with low frequencies $f$ are performed. A dynamical consideration of the compensation unit is not discussed in this paper. To reduce the influence of the voltage profile of the applied voltage $U_{\text {piezo }}$ on the maximum error $e_{\max }$, a reference profile is created. The reference profile follows hysteresis loops of various amplitudes $\hat{U}_{\text {piezo }}$ to allow conclusions between different hysteresis loops and the maximum error $e_{\max }$. The presented influences are examined by conducting several runs with different configurations.

\subsection{EXPERIMENTAL PROCEDURE}

At the beginning of each run, three hysteresis loops with an amplitude $\hat{U}_{\text {piezo }}=600 \mathrm{~V}$ are performed. Based on the averaged trajectories of the second and third loops, the hysteresis operator is created. Each run is carried out with a sinusoidal excitation with a freq- 
uency $f=1 \mathrm{~Hz}$, shown in Fig. 5. The voltage profile of the sinusoidal excitation starts at voltage $U_{\text {piezo }}=0 \mathrm{~V}$ with an amplitude $\hat{U}_{\text {piezo }}=600 \mathrm{~V}$, then the amplitude is gradually reduced to $\hat{U}_{\text {piezo }}=200 \mathrm{~V}$. The second part of the profile starts at voltage $U_{\text {piezo }}=600 \mathrm{~V}$ with an amplitude $\hat{U}_{\text {piezo }}=200 \mathrm{~V}$. Here the amplitude is gradually increased to $\hat{U}_{\text {piezo }}=600 \mathrm{~V}$. For reason of comparability, the time course is divided into six sections $i$, marked in Fig. 5.

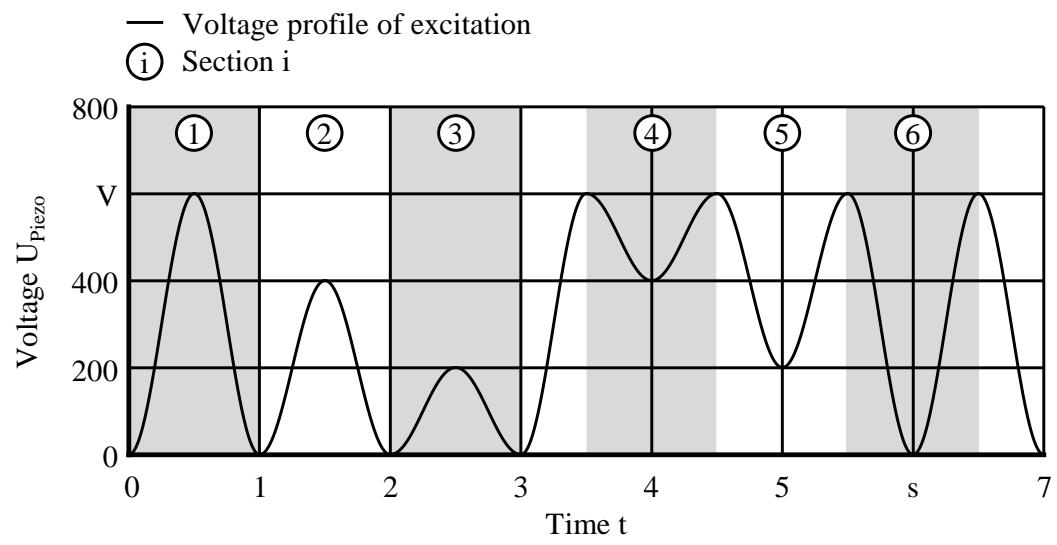

Fig. 5. Voltage profile of the applied voltage $U_{\text {piezo }}$ as excitation for the experimental investigation

To examine the influence of the preload force $F_{\mathrm{PF}}$, the preload stiffness $k_{\mathrm{PS} 2}$ and the mass $m_{w}$ on the size of the maximum error $e_{\max }$, in total four configurations of the compensation unit are tested, see Table 2. In the first configuration, the preload spring PS2 is installed such that a preload force $F_{\mathrm{PF}} \approx 5 \mathrm{kN}$ and a preload stiffness $k_{\mathrm{PS} 2}=12 \mathrm{~N} / \mu \mathrm{m}$ appear and the platform is loaded without any mass $m_{w}$. The second configuration corresponds to the first but loaded with the mass $m_{w}=14 \mathrm{~kg}$. In the third configuration, the mass $m_{w}$ is removed again and the preload force $F_{\mathrm{PF}}$ is reduced to a minimum by releasing the preload spring PS2, while the preload stiffness $k_{\mathrm{PS} 2}=12 \mathrm{~N} / \mu \mathrm{m}$ is still applied. In the fourth configuration, the preload spring PS2 is completely removed so neither a preload force $F_{\mathrm{PF}}$, a preload stiffness $k_{\mathrm{PS} 2}$ nor a mass $m_{w}$ is acting. Ten runs are performed and evaluated for each configuration. Thus, the database contains a total of forty runs. The mean value $\overline{e_{i}}$ of the maximum error $e_{\max }$ for each section $i$ over ten runs for each configuration are calculated and thus a comparison of the configurations is possible.

Table 2. Configurations of the compensation unit

\begin{tabular}{|c|c|c|c|}
\hline Configuration & Preload force $F_{\mathrm{PF}}$ & Preload stiffness $k_{\mathrm{PS} 2}$ & Mass $m_{w}$ \\
\hline 1 & $5 \mathrm{kN}$ & $12 \mathrm{~N} / \mu \mathrm{m}$ & $0 \mathrm{~kg}$ \\
\hline 2 & $5 \mathrm{kN}$ & $12 \mathrm{~N} / \mu \mathrm{m}$ & $14 \mathrm{~kg}$ \\
\hline 3 & $0 \mathrm{kN}$ & $12 \mathrm{~N} / \mu \mathrm{m}$ & $0 \mathrm{~kg}$ \\
\hline 4 & $0 \mathrm{kN}$ & $0 \mathrm{~N} / \mu \mathrm{m}$ & $0 \mathrm{~kg}$ \\
\hline
\end{tabular}

\section{RESULTS AND DISCUSSION}

First, the resolution $d$ of a force estimation is determined without the use of a hysteresis operator, according to Equation 2. The maximum height $h_{H}=8.96 \mu \mathrm{m}$ of the hysteresis curve 
leads to a resolution $d \leq 1,389 \mathrm{~N}$. The results using the hysteresis operator are discussed below. Figure $6 \mathrm{a}$ and $6 \mathrm{~b}$ show the time signal of the measured and estimated displacement $x_{p}$ and Fig. 6c and 6d show the measured and estimated hysteresis curves for one run in configuration 1. Figure 7 shows the mean value $\overline{e_{i}}$ of the maximum error $e_{\max }$ for all configurations in each section $i$ and additionally mean value $\overline{e_{5}}$ together the standard deviation $s d_{5}$ of section $i=5$. Figure 7 shows that configuration 3 provides lower values for the averaged maximum error $\overline{e_{i}}$ than configuration 4 , i.e. in the absence of a preload force $F_{\mathrm{PF}}$, the presence of a preload stiffness $k_{\mathrm{PS} 2}$ leads to an improvement. Configuration 3 shows the best result in section $1 \leq i \leq 3$, whereas in section $4 \leq i \leq 6$ the values for the averaged maximum error $\overline{e_{i}}$ are above configuration 1 . The overall lowest values for the averaged maximum error $\bar{e}$ in all sections $i$ with the mass $m_{w}=0 \mathrm{~kg}$ are achieved in configuration 1 . In comparison to configuration 1, configuration 2 shows a slight reduction in the averaged maximum error $\overline{e_{i}}$ in all sections $i$. For configuration 1 the averaged maximum error is $\bar{e}=0.50 \mu \mathrm{m}$. According to Equation 3 and considering the stiffness $k_{p}=155 \mathrm{~N} / \mu \mathrm{m}$, this results in a resolution $d \leq 78 \mathrm{~N}$.

The results show that it is possible to determine an effecting process force $F_{p}$ using the presented force estimation approach. Since the compensation unit is loaded with different workpieces and thus different masses $m_{w}$ during production operations, the force estimation should not get worse under load with a mass $m_{w}$. This is shown by the measurements in configuration 2, where the mass $m_{w}$ has a positive effect on the maximum error $e_{\max }$.
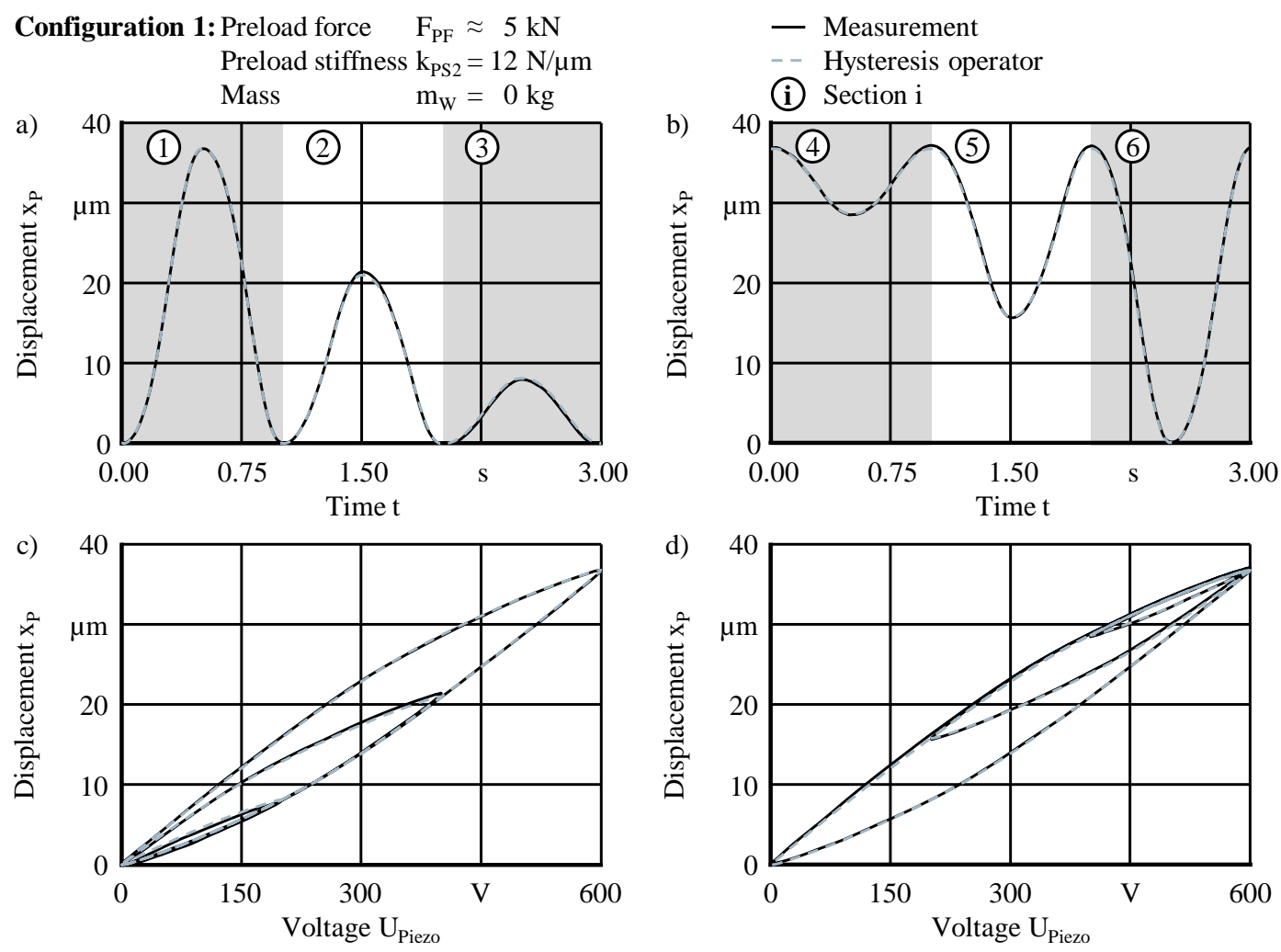

Fig. 6. Measured and estimated displacement $x_{p}$ of the platform resulting from a sinusoidal excitation: a) time signal for section $1 \leq i \leq 3$, b) time signal for section $4 \leq i \leq 6$, c) hysteresis curve for section $1 \leq i \leq 3, \mathrm{~d})$ hysteresis curve for section $4 \leq i \leq 6$ 


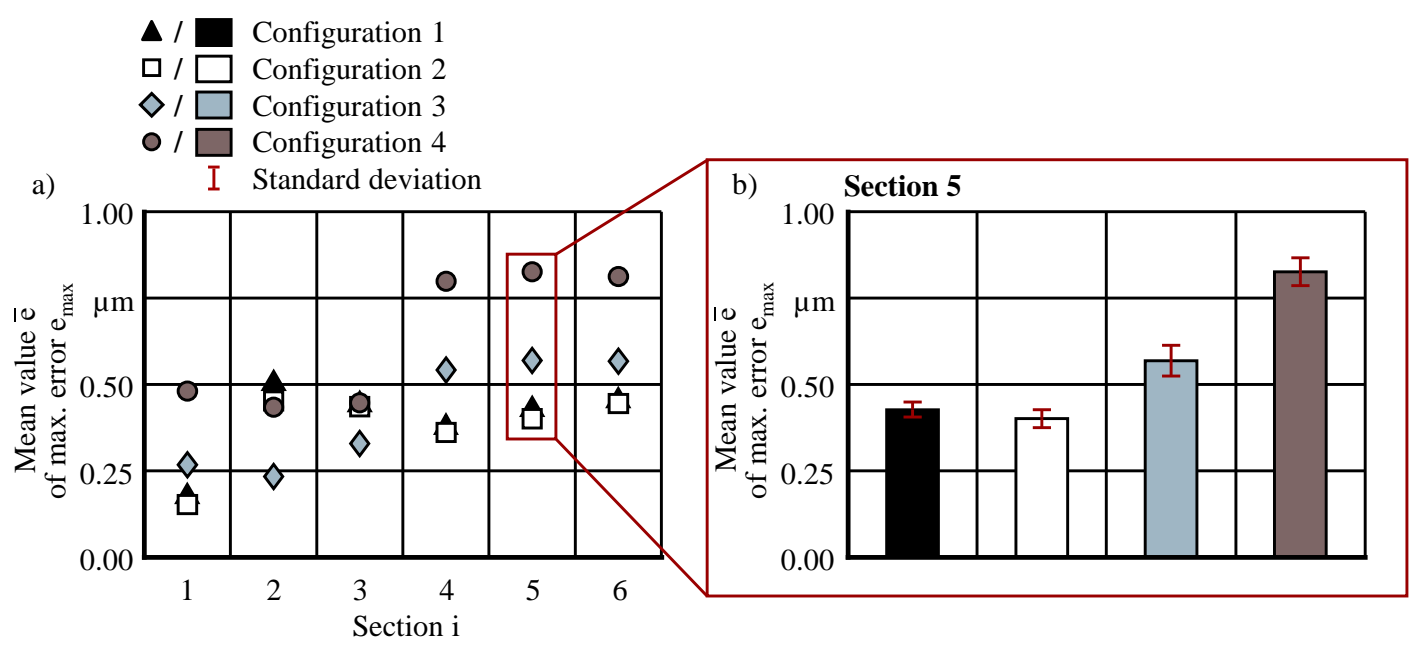

Fig. 7. Experimental results on mean value $\overline{e_{i}}$ of the maximum error $e_{\max }$ for all configurations: a) Section $1 \leq i \leq 6$, b) Section $i=5$ with standard deviation

The achievable resolution $d \leq 78 \mathrm{~N}$ is determined by the stiffness $k_{p}$ of the platform and the maximum error $e_{\max }$. It can be assumed that the maximum error $e_{\max }$ can be further reduced by improving the hysteresis operator [16] and by the implementation of an operator to compensate the creep behavior. One possibility to reduce the stiffness $k_{p}$ of the platform is to decrease the stiffness $k_{\mathrm{FH}}$ of the flexure hinges by changing their geometry. At the same time, it is important to ensure that the stiffness of the platform in the vertical direction and its torsional stiffness remain high. For that, appropriate constructive solutions must be developed. Another possibility is the use of piezoelectric actuators with a lower stiffness $k_{\text {piezo. }}$. The use of the piezo actuators PSt 1000/35/200 VS45 made by Piezosystem Jena GmbH, Jena, Germany, with a stiffness $k_{\text {piezo }}=150 \mathrm{~N} / \mu \mathrm{m}$ for example would reduce the stiffness $k_{p}$ of the platform by $17 \%$. The stiffness $k_{C}$ of the connection between actuator and platform also has a major influence on the stiffness $k_{p}$, but a reduction would lead to a reduced displacement $x_{p}$ of the platform and a deterioration of the compensation unit's dynamic behavior.

\section{CONCLUSION}

The aim of the present work was the implementation of a force estimation in a piezoelectric compensation unit without the use of external force sensors and the determination of its resolution $d$. This was done by comparing the voltage control of the piezoelectric actuators with the measured values of the displacement sensors. This approach resulted in a resolution $d \leq 1,389 \mathrm{~N}$ of the force estimation. To face strong nonlinear hysteresis effects of the actuators, a hysteresis operator was implemented. Thereby an improvement of the resolution about 94\% down to $d \leq 78 \mathrm{~N}$ was achieved. Different options for further improvements are discussed in Section 5. In addition to the existing deviations of the hysteresis operator, limiting factors are the creep behavior of the actuators, the systems noise as well as a high stiffness $k_{p}$ of the platform. Future works will focus on the reduction of these influences to enable the force estimation for the online compensation of machine tool deflections. 


\section{REFERENCES}

[1] SPUR G., 1996, Die Genauigkeit von Maschinen: Eine Konstruktionslehre, Hanser, Munich, Germany.

[2] SCHLEIPEN M., LÜDER A., SAUER O., FLATT H., JASPERNEITE J., 2015, Requirements and Concept for Plug-and-Work: Adaptivity in the context of Industry 4.0, Automatisierungstechnik, 63/10, 801-820.

[3] HABIBI M., AREZOO B., VAHEBI NOJEDEH M., 2011, Tool Deflection and Geometrical Error Compensation by Tool Path Modification, International Journal of Machine Tools and Manufacture, 51/6, 439-449.

[4] DENKENA B., MÖHRING H.-C., WILL J.C., 2007, Tool Deflection Compensation with an Adaptronic Milling Spindle, in: International Conference on Smart Machining Systems (ICSMS), Gaithersburg, USA.

[5] HEO S., LEE M., KIM S.H., LEE W., MIN B.K., 2015, Compensation of Tool Deflection in Micromilling Using Workpiece Holder Control Device, Int. J. Precis. Eng. Manuf., 16/6, 1205-1208.

[6] DENKENA B., BOUJNAH H., 2018, Feeling Machines for Online Detection and Compensation of Tool Deflection in Milling, CIRP Annals, 67/1, 423-426.

[7] KALLAGE F., 2007, Einsatz Magnetischer Aktor- und Führungseinheiten zur Erhöhung der Bahngenauigkeit von Hochgeschwindigkeitsfräsmaschinen, Berichte aus dem IFW, PZH Produktionstechnisches Zentrum GmbH, Garbsen, Germany.

[8] LISELI J.B., AGNUS J., LUTZ P., RAKOTONDRABE M., 2020, An Overview of Piezoelectric Self-Sensing Actuation for Nanopositioning Applications: Electrical Circuits, Displacement, and Force Estimation, IEEE Trans. Instrum. Meas., 69/1, 2-14.

[9] RAKOTONDRABE M., IVAN I.A., KHADRAOUI S., LUTZ P., CHAILLET N., 2015, Simultaneous Displacement/Force Self-Sensing in Piezoelectric Actuators and Applications to Robust Control, IEEE/ASME Trans. Mechatron., 20/2, 519-531.

[10] IVAN I.A., RAKOTONDRABE M., LUTZ P., CHAILLET N., 2009, Current Integration Force and Displacement Self-Sensing Method for Cantilevered Piezoelectric Actuators, The Review of Scientific Instruments, 80/12.

[11] IVAN I.A., RAKOTONDRABE M., LUTZ P., CHAILlET N., 2009, Quasistatic Displacement Self-Sensing Method for Cantilevered Piezoelectric Actuators, The Review of Scientific Instruments, 80/6.

[12] KAWAMATA A., KADOTA Y., HOSAKA H., MORITA T., 2008, Self-Sensing Piezoelectric Actuator Using Permittivity Detection, Ferroelectrics, 368/1, 194-201.

[13] ISLAM M., SEETHALER R., MUMFORD D., 2011, Hysteresis Independent On-Line Capacitance Measurement for Piezoelectric Stack Actuators, $24^{\text {th }}$ Canadian Conference on Electrical and Computer Engineering (CCECE), IEEE, 1149-1153.

[14] ZARIF MANSOUR S., SEETHALER R., 2018, Simultaneous Quasi-Static Displacement and Force Self-Sensing of Piezoelectric Actuators by Detecting Impedance, Sensors and Actuators A: Physical, 274, 272-277.

[15] KIANINEJAD K., THOM S., KUSHWAHA S., UHLMANN E., 2016, Add-on Error Compensation Unit as Sustainable Solution for Outdated Milling Machines, Procedia CIRP, 40, 174-178.

[16] RAKOTONDRABE M., 2012, Classical Prandtl-Ishlinskii Modeling and Inverse Multiplicative Structure to Compensate Hysteresis in Piezoactuators, American Control Conference, Montréal, Canada. 